Faculté de pédagogie, département

de Curricula et de Méthodologie.

\title{
Effet de l'apprentissage collaboratif en ligne pour améliorer la compréhension orale du FLE chez les futurs-enseignants à la faculté de pédagogie
}

\author{
Préparé par \\ Abd El-Monème Samir Alsaka \\ Maître-assistant du FLE à l'institut supérieur \\ du tourisme et d'Hôtellerie, d'Alexandrie \\ Prof. Azza Abdel Razek Abd rabeh \\ Professeur émérite de curricula et de méthodologie \\ du FLE, Faculté de pédagogie \\ Université de Tana \\ Prof. Adel Tawfiq Ibrahim \\ Professeur de curricula et de méthodologie \\ du FLE et le doyen précèdent de la Faculté de \\ pédagogie, Université d'Al Sadat-Ville
}

\section{Prof. Mamdouh Mohammed Abd El-Maboud \\ Professeur de curricula et de méthodologie de \\ La science, et le doyen de la Faculté de \\ Pédagogie, Université \\ D'Al Sadat-Ville.}




\section{RÉSUMÉ EN FRANÇAIS}

La recherche actuelle vise à améliorer les compétences de la compréhension orale du FLE, chez les étudiants de la faculté de pédagogie université d'Al-Sadat. En ayant recours à l'apprentissage collaboratif en ligne, le chercheur a utilisé un environnement virtuel. Il a présenté les modules à travers un site collaboratif. L'expérimentation a duré trois mois. Un seul groupe expérimental a participé à cette expérimentation.

L'objectif général de la recherche est d'attirer l'attention au rôle des TICE dans l'apprentissage du FLE en général et surtout dans l'amélioration des compétences de la compréhension du FLE.

Les résultats de la présente recherche montrent l'intérêt d'intégrer les TICE dans l'apprentissage/enseignement du FLE afin d'améliorer les compétences de la compréhension orale du FLE chez les étudiants de la faculté de pédagogie université d'Al-Sadat.

\section{ملخص باللغة العربية}

يهدف البحث الحالي إلى تحسين مهار ات الفهم الثفهي للغة الفرنسية, لدى الطلاب المعلمين بكلية التربية, جامعة السادات, باستخدام استر اتيجية التعليم التشاركى الألكترونى, حيث استخدم الباحث بيئة افتر اضية, استطاع من خلالها تقديم البرنامج الألكترونى من خلال موقع تشاركى. استغرق التطبيق ثلاثة أشهر. شاركت مجمو عة تجريبية واحدة فقط في هذه التجربة.

$$
\text { اللغةيهدف البحث الحالى أيضا إلى تركيز الانتباه إلى دور تكنولوجيا المعلومات و الاتصالات في تعلم }
$$

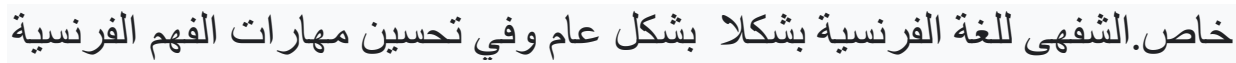

تظهر نتائج هذا البحث الفائدة الكبيرة من دمج تكنولوجيا المعلومات والاتصالات في تعليم وتعلم اللغة

الفرنسية من أجل تحسين مهار ات الفهم الثفهي للغة الفرنسية لطلاب كلية التربية, جامعة السادات. 
Nous vivons à une époque de profondes mutations où les technologies de l'information et la communication (TIC) jouent un rôle de plus en plus central dans tous les domaines de la vie. Elles ont une influence croissante sur l'évolution des sociétés et affectent de façon significative les dimensions économique, sociale et éducative.

Les TIC peuvent effectivement favoriser la collaboration et la rendre plus efficace. L'accès facile à un ordinateur ou un portable en réseau permettra de poursuivre les échanges amorcés sur place ou à distance, de collaborer à un même projet et de recevoir l'avis de collègues ou d'experts. C'est dire que la technologie peut favoriser une ouverture, un échange, une collaboration et un partage de connaissances entre des collègues poursuivant les mêmes objectifs. Cette collaboration entre apprenants peut aussi être bénéfique. (Allaire \& Lusignan. 2019:17)

La collaboration en ligne est de plus en plus présente dans la FAD au niveau universitaire pour la dynamique qu'elle crée et les possibilités que peut offrir le groupe collaboratif en tant que catalyseur de l'apprentissage. Une grande partie des FAD sont construites sur le modèle socioconstructiviste et sont basées sur l'apprentissage collaboratif où « les apprenants travaillent ensemble en vue de la réalisation d'un but commun conciliant leurs intérêts et leurs objectifs personnels ». (Henri \& Cayrol, 2008:33)

Dans l'acquisition de la langue étrangère, la compréhension précède l'expression. Avant de pouvoir formuler et transmettre un message oral, l'étudiant doit avoir entendu et compris la langue. Il doit avoir été baigné dans un milieu linguistique riche et varié. Plus l'étudiant sera récepteur de messages significatifs, plus il sera en mesure de s'approprier la langue. L'enseignant a donc un rôle important à jouer par rapport à l'écoute et la lecture. Les enjeux scolaires de la compréhension sont apparus de plus en plus évidents à l'ensemble du corps social. (Franc. P .2018:18)

À savoir que dans cette présente recherche on met l'accent sur l'importance de l'apprentissage collaboratif en ligne pour apprendre la compréhension orale du FLE. La compréhension orale qui est à la base d'une grande partie des apprentissages, est une activité mentale de construction du sens qui peut s'exercer dans tous les registres. (Vendergrift. 2012:39)

La compréhension orale est une des étapes les plus fondamentales de la communication et dans l'acquisition d'une langue étrangère. L'écoute dans des situations authentiques permet à l'apprenant de se familiariser avec les sons de la langue non maternelle. Elle permet de présenter à l'apprenant des discours plutôt que des phrases. Elle amène l'apprenant à s'appuyer sur les éléments, tant linguistiques que non linguistiques, pour comprendre le message oral. (Ferroukhi, k, 2009:1)

En ce sens on peut révéler que, l'objectif de notre recherche est d'une part de développer les compétences de la compréhension orale du FLE chez les futursenseignants et d'autre part de tenter et déterminer les effets de ce dispositif de formation, ou, plus précisément, les effets d'une utilisation particulière de la démarche collaborative en ligne sur les apprentissages et les intentions de changement de pratique d'un groupe de futurs-enseignants. 
Cette présente recherche a été inspirée des études antérieures élaborées dans le domaine de la didactique de FLE afin d'étudier et de renforcer les compétences de la compréhension l'oral.

D'une étude pilote: Pendant les cours pratiques du micro enseignement aux étudiants de la troisième année, section de français à la faculté de pédagogie, université d'Elsadat, Le chercheur a remarqué à l'aide une collègue, le faible niveau des compétences de la compréhension orale, chez les étudiants.

\section{Problématique de la recherche:}

La problématique de la présente recherche réside dans la question principale suivante:

«Quel est l'effet d'un dispositif collaboratif en ligne pour développer la compréhension orale en FLE chez les futurs-enseignants de la faculté de pédagogie, université d'Al Sadat? »

Pour répondre à la question principale, il est nécessaire de poser des questions sous-jacentes issues :

1. Quelles sont les compétences en compréhension orale, nécessaires aux futurs enseignants de FLE?

2. Comment peut-on développer les compétences en compréhension orales chez des futurs enseignants de FLE?

3. Quels sont les effets d'un dispositif d'apprentissage collaboratif en ligne pour développer les compétences en compréhension orales chez les futurs enseignants de FLE?

\section{Hypotheses de la recherche:}

"Il n'y a pas de différences statistiquement significatives entre les moyennes des notes des futurs-enseignants aux pré-post tests des compétences de la compréhension orale.»

"Il y a de différences statistiquement significatives entre les moyennes des notes des apprenants aux pré-post tests des compétences de la compréhension orale en FLE en faveur du post-test. »

3. Importance de l'étude:

1. Faire évoluer l'auto apprentissage qui est important pour développer les compétences de la compréhension orale des enseignants du FLE tout au long de la vie.

2. Engager les futurs enseignants dans une nouvelle expérience (l'apprentissage collaboratif en ligne) pour partager leurs connaissances avec autrui, cette optique développe chez eux les principes du travail en équipe, le respect de l'autre, la responsabilité, l'autonomie et etc.

3. Améliorer les programmes de formation initiale des enseignants (es) du FLE, et de contribuer à la professionnalisation du dispositif de formation.

4. Objectifs de la recherche:

1. Déterminer les compétences de la compréhension orale nécessaires aux futurs 
2. Évaluer le degré de la maîtrise des compétences de la compréhension oralechez les futurs enseignants du FLE.

3. Déterminer les effets d'un dispositif basé sur l'apprentissage collaboratif en ligne au développement des compétences de la compréhension orale chez les futurs enseignants du FLE.

4. Sensibiliser à l'emploi de l'informatique.

\section{Délimites de la recherche:}

- À un échantillon ciblé de 12 futurs-enseignants de FLE (les étudiants (es) de la troisième année) section de français à la faculté de Pédagogie, Université d'Elsadat

- Au développement de quelques compétences de la compréhension orale chez les futurs enseignants de FLE.

- À l'élaboration d'un dispositif proposé concernant les compétences de la compréhension du FLE.

- À la préparation d'un environnement électronique dans un espace approprié pour les futurs-enseignants de la faculté de pédagogie d'Alsadat.

6. Méthodologie de recherche: À la lueur des objectifs de cette recherche et la nature des données nécessaires pour atteindre ces objectifs, le chercheur a utilisé :

- La méthode descriptive

- La méthode experimentale

\section{Outils de la recherche:}

1. Test de placement.

2. Les pré-post tests des modules proposés.

3. Fiche d'évaluation des compétences de la compréhension orale.

4. Grille d'évaluation des compétences de la compréhension orale.

5. Un portfolio pur chaque étudiants.

\section{Procédures de la recherche:}

1. Présenter une étude théorique traitant deux axes essentiels :

- Axe (1):l'apprentissage collaboratif en ligne.

- Axe (2): la compréhension orale du FLE.

2. Choisir un échantillon ciblé.

3. Elaborer les outils de l'étude :

- Test de placement

- Pré-post tests des modules proposés.

- Fiche d'évaluation des compétences de la compréhension orale

- Grille d'évaluation des compétences de la compréhension orale.

4. Construire le dispositif éducatif.

5. Appliquer le pré-test concernant les compétences de la compréhension de FLE.

6. Enseigner les modules visés du dispositif proposé en ligne. 
7. Appliquer l'outil d'évaluation.

8. Appliquer le post-test concernant les compétences de la compréhension orale de FLE.

9. Analyser et interpréter les résultats.

10. Présenter les recommandations de l'étude et les suggestions.

8. Terminologies de la recherche: Le chercheur défini la compréhension comme l'aptitude qui résulte de la mise en œuvre de processus cognitifs, qui permet au futurenseignant d'accéder au sens d'un texte qu'il écoute (compréhension orale) ou qu'il lit (compréhension écrite). Il faut distinguer l'écoute et la lecture qui sont des pratiques volontaires, des processus cognitifs, largement involontaires.

Le chercheur définit l'apprentissage collaboratif en ligne comme une démarche active et centrée sur le futur-enseignant. Au sein d'un groupe et dans un environnement approprié, le futur-enseignant exprime ses idées, articule sa pensée, développe ses propres représentations, élabore ses structures cognitives et fait une validation sociale de ses nouvelles connaissances.

\section{Le cadre théorique}

\section{Introduction :}

L'éducation est aujourd'hui soumise à un changement de contexte: la technologie est maintenant omniprésente, et les sciences de l'apprentissage évoluent. Ce changement porte plus particulièrement sur les rapports qu'un apprenant entretient avec le savoir, sur le temps, sur les espaces éducatifs et sur l'importance du collectif dans l'apprentissage. Ainsi, les mutations en cours viennent interroger le rôle et la formation des futursenseignants qui, d'une part, bénéficient de nouvelles opportunités en ce qui concerne la pédagogie centrée sur l'apprenant, mais, d'autre part, doivent aussi affronter de nouveaux défis. (Guichon, N.2015:22)

L'apprentissage en ligne désigne une méthode d'acquisition d'un savoir ou de construction de connaissance utilisant des interactions (acteur-acteur ou acteursressources) relayées par un système télématique (électronique, informatique connecté par réseau). L'apprentissage électronique peut avoir lieu à distance (en ligne), en classe (hors ligne et/ou en ligne) ou les deux. L'apprentissage en ligne est une spécialisation de l'apprentissage à distance (ou formation à distance). (Duquesnoy, M. 2018:8)

\section{$\underline{1}^{\mathrm{er}}$ axe : L'apprentissage collaboratif en ligne.}

Pour Désy, l'apprentissage collaboratif en ligne constitue un terme générique chapeautant un ensemble de principes et de techniques pédagogiques émergeant des théories cognitivistes et définissant l'apprentissage comme un construit social qui résulte d'une activité de collaboration en ligne. (Dussault-Désy, 2010:43)

Le chercheur définit l'apprentissage collaboratif en ligne comme une démarche active et centrée sur le futur-enseignant. Au sein d'un groupe et dans un environnement approprié, le futur-enseignant exprime ses idées, articule sa pensée, développe ses propres représentations, élabore ses structures cognitives et fait une validation sociale 
démarche collaborative en ligne reconnaît les dimensions individuelle et collective de l'apprentissage, encourage l'interaction et exploite les cognitions réparties au sein de l'environnement.

En reprenant les propositions de Springer, on peut définir les bénéfices de l'apprentissage collaboratif en ligne de la manière suivante :

- Les acteurs ont/se donnent des tâches complexes à accomplir avec un but clairement défini.

- Ils prennent ensemble des décisions sur la façon de procéder.

- Ils ne font pas forcément tous la même chose au même moment.

- Ils ont accès à un grand nombre de ressources.

- Ils traitent une grande quantité d'informations authentiques.

- Ils interagissent entre eux en ligne, mais aussi avec des personnes externes (experts, membres de la communauté, etc.).

- Ils sont engagés dans un processus de découverte / construction des connaissances.

- Ils réfléchissent sur leurs actions et sur les ressources mobilisées.

- Ils communiquent et partagent leur savoir en ligne.

- Ils s'autoévaluent en ligne tout au long du processus pour améliorer le résultat.

- Ils développent de nouvelles compétences tout en s'impliquant dans l'action, ces compétences sont à la fois individuelles, sociales, transversales et spécifiques. (Springer \& Koenig-Wisniewska.2019:14.SI)

Pour assurer le succès de l'apprentissage collaboratif en ligne, Henri et Lundgren propose cinq conditions:

- Un nombre, une dispersion et une diversification suffisants de futursenseignants en ligne pour former assez de petits groupes suffisamment diversifiés et dispersés tout en permettant la participation de chacun à l'ensemble des activités.

- Des moyens de participation effective à l'interaction textuelle asynchrone de chaque apprenant : disponibilités de temps, moyens et habiletés technologiques et capacités d'expression textuelle requises.

- Une motivation des futurs-enseignants pour le contenu de la formation et pour la collaboration entre personnes différentes ayant un but commun.

- Une cohésion et une productivité suffisantes du groupe virtuel résultant de leur motivation, de l'objectif commun, de l'activité et du dispositif virtuel proposés.

- Une animation adroite par un animateur adaptant les apprenants à cet environnement virtuel, stimulant leur motivation, organisant et coordonnant leur participation, valorisant leurs apports, liant au contenu de la formation et les 
synthétisant dans l'optique de l'activité à réaliser. (Henri et Lundgren.2001:7678)

Schrage ajoute, pour comprendre l'importance de la conversation dans le processus de collaboration, que les collaborateurs se réunissent pour échanger, pour partager leurs idées et leur vision des choses, pour élaborer des hypothèses, pour discuter et s'entendre sur l'orientation à donner au travail en ligne.(Schrage:2011:59)

Pour Arpin les environnements d'apprentissage collaboratif virtuels sont actuellement en grande demande dans les milieux de l'éducation, de la formation et du perfectionnement. On y voit une réponse prometteuse à la demande pressante de modèles d'apprentissage adaptables à différents profils de futurs-enseignants et assez souples pour lever les contraintes de temps, d'espace et d'accès aux ressources. (Arpin, L. et Carpa, L.2010:23)

Bien que le rôle de l'enseignant demeure crucial. Ce rôle doit être différent de celui d'une organisation traditionnelle de la classe. L'enseignant doit accepter de ne plus être la source unique de savoir. Mais une personne qui vise à mettre en place les conditions favorables à l'apprentissage. (Burge, 2014:34)

Chaque membre, dans un groupe collaboratif doit:

- Avoir des tâches complexes à accomplir avec un but clairement défini.

- Prendre ensemble des décisions sur la façon de procéder.

- Ne pas faire forcément tous la même chose au même moment.

- Avoir accès à un grand nombre de ressources.

- Traiter une grande quantité d'informations authentiques.

- Interagir entre eux, mais aussi avec des personnes externes (experts, membres de la communauté, etc.).

- Engager dans un processus de découverte / construction des connaissances.

- Réfléchir sur leurs actions et sur les ressources mobilisées.

- Communiquer et partager leur savoir.

- S'autoévaluer tout au long du processus pour améliorer le résultat.

- Développer de nouvelles compétences tout en s'impliquant dans l'action. (Springer \& Koenig-Wisniewska.2019:14)

Dans cette optique, pour réussir au développement de la compréhension orale de FLE à travers l'apprentissage collaboratif en ligne, on va présenter un programme proposé en ligne, qui se compose de modules et de séances pour améliorer les compétences de la compréhension orale.

\section{$2^{\mathrm{e}}$ axe : La compréhension du FLE.}

La compréhension orale est une compétence qui vise à faire acquérir progressivement à l'apprenant des stratégies d'écoute premièrement et $d$ compréhensions d'énoncé à l'orale deuxièmement. Il ne s'agit pas d'essayer de tout faire comprendre une définition pour chaque mot, il est question au contraire de former les apprenants à devenir plus sur d'eux plus autonomes progressivement. C'est-à-dire La compréhension orale est une activité constitue la première compétence qui doit être 
apprentissage d'une langue étrangère par ce qu'elle vise à faire acquérir progressivement à l'apprenant des stratégies d'écoute au préalable. Ensuite, la compréhension d'énoncé à l'oral en second lieu. (Cherak, R. 2019:15)

Le chercheur défini la compréhension orale comme l'aptitude qui résulte de la mise en œuvre de processus cognitifs, qui permet au futur-enseignant d'accéder au sens d'un texte qu'il écoute. Il faut distinguer l'écoute qui est des pratiques volontaires, des processus cognitifs, largement involontaires. Dans ce cas, il est indispensable de fournir au futur- enseignant les moyens nécessaires pour développer ses capacités en compréhension orale

Selon Michelas les aspects linguistiques de la compréhension orale comprennent cinq éléments à savoir: la discrimination auditive, l'intonation, l'accentuation, les connaissances lexicales et les connaissances grammaticales. (Michelas, A. 2019:30)

En utilisant la technique de la réflexion à haute voix, Mackay (2002) a analysé les stratégies d'écoute de l'apprenant de niveau collégial en anglais langue étrangère. Cette expérience lui a permis de déterminer quatre stratégies fréquemment utilisées par les auditeurs les plus compétents.

Si l'on veut faciliter l'apprentissage, la segmentation de la tache de compréhension orale en quelques étapes demeure essentielle à cet effet les didacticiens, Cornaire (2006), et Marchi ( 2010), proposent une démarche de trois étapes: la pré écoute, l'écoute et la poste écoute.

La pré écoute est la phase pendant laquelle, on amène les futurs-enseignants à anticiper le contenu du texte qu'ils vont entendre. L'enseignant doit s'assurer que les apprenants ont quelques connaissances sur le sujet à étudier. Si tel n'était pas le cas, il faut alors leur en fournir ou leur demander d'aller se renseigner sur la question.

Pour Lhote, l'écoute c'est l'étape où les futurs-enseignants tentent de dégager le sens global du texte en s'appuyant sur tous les indices linguistiques et non linguistiques. C'est le moment où ils vérifient les hypothèses qu'ils avaient proposées dans la phase de pré écoute. Pendant la première écoute, l'apprenant est appelé à comprendre la situation pour saisir le cadre dans lequel le texte prend place. Il est très utile de se préparer à reconnaître le contour situationnel à l'intérieur duquel se déroulent les évènements. (Lhote, F.2005:70-71)

Pour Marchi, la poste écoute c'est l'étape au cours de laquelle les apprenants évaluent et critiquent autant ce qu'ils ont compris selon les stratégies qu'ils ont utilisées. C'est la phase où ils partagent leurs impressions et expriment leurs sentiments. Ils décident avec l'enseignant quelle suite ils veulent donner à l'activité; écouter ou regarder d'autres œuvres, lire des livres sur le même sujet, chercher des informations supplémentaires, etc. Il est également important pendant cette phase de réinvestir les compétences acquises dans une tâche réelle. (Marchi, B.2010: 67)

De tout ce qui précède nous pouvons remarquer le rôle vital de la compréhension orale, dans l'apprentissage d'une langue étrangère, de sorte qu'on n'arrive plus à acquérir une langue sans le perfectionnement de cette compétence. Mais à fin d'entrainer les étudiants à la compréhension orale, il 
faut adopter une des méthodes ou une certaine démarche déterminée, en profitant des TIC, qui vise à améliorer cette compétence. C'est pour cette raison que notre étude va essayer d'améliorer cette compétence chez les étudiants de la troisième et de la quatrième année à la faculté de pédagogie en employant un programme proposé basé sur l'apprentissage collaboratif en ligne.

\section{Méthodologie de la recherche}

La problématique de la présente recherche réside dans la question principale suivante: «Quel est l'effet d'un dispositif collaboratif en ligne pour développer la compréhension orale en FLE chez les futurs-enseignants de la faculté de pédagogie, université d'Al Sadat? »

Pour répondre à la question principale, il est nécessaire de poser des questions sous-jacentes issues :

1. Quelles sont les compétences en compréhension orale, nécessaires aux futurs enseignants de FLE?

2. Comment peut-on développer les compétences en compréhension orales chez des futurs enseignants de FLE?

3. Quels sont les effets d'un dispositif d'apprentissage collaboratif en ligne pour développer les compétences en compréhension orales et écrites chez les futurs enseignants de FLE?

Le chercheur divise cette partie en deux axes dont le premier concerne les démarches suivies pour préparer les outils de la recherche, et le deuxième porte sur la démarche qu'on a suivie pour élaborer et construire le dispositif éducatif proposé aussi bien que la procédure de l'expérimentation.

- Choisir l'échantillon:

Le chercheur a choisi les futurs-enseignants de la troisième année, section de français, faculté de pédagogie d'Al-Sadat, de l'année universitaire 2019-2020 pour les raisons suivants :

Nous avons utilisé un seul groupe expérimental pour les raisons suivantes:

- Le nombre des étudiants de la troisième année après exclure l'échantillon du test de placement, ne permet pas l'emploi de plus qu'un groupe,

- Eviter la transmission des compétences du groupe expérimental au groupe témoin,

- Avoir difficulté d'étudier de la manière traditionnelle avec un groupe témoin à cause de COVID-20 et s'arrêter l'étude traditionnelle dans l'Etat.

En effet, le chercheur a choisi son échantillon selon les considérations suivantes :

- Le niveau de leur acquis linguistique préalable du français, langue étrangère, 
- Le niveau de leur utilisation de l'ordinateur où ils ont étudié dans leur curricula de deuxième année une matière concernant les compétences informatiques et comment utiliser l'ordinateur. Cette matière est intitulée "Technologie et moyens d'apprentissage",

- les membres de l'échantillon se caractérise par la curiosité de s'ouvrir au monde et de rencontrer d'autres cultures

- L'âge des membres de l'échantillon vacille entre (19-21).

- La période de transition chez les apprenants de la base théorique à l'usage et à la pratique, ainsi que c'est la période pendant laquelle les apprenants peuvent se former et acquérir des compétences de la compréhension orale et écrite.

Afin de mener à bien un programme visant à développer quelques compétences de la compréhension du FLE, le chercheur a choisi 12 étudiants et étudiantes. Ils ont environ 19 à 21 ans.

\section{(Axe 1). Outils de la recherche.}

Pour répondre aux hypothèses suivantes :

\section{Hypothèse nulle :}

"Il n'y a pas de différences statistiquement significatives entre les moyennes des notes des futurs-enseignants aux pré-post tests des compétences de la compréhension orale.»

\section{Hypothèse dirigée:}

"Il y a de différences statistiquement significatives entre les moyennes des notes des apprenants aux pré-post tests des compétences de la compréhension orale en FLE en faveur du post-test. »

\section{Le chercheur a donc élaboré les outils suivants :}

* Un test de placement,

* Une fiche d'évaluation des compétences de la compréhension orale de FLE,

* Une grille d'évaluation de la compréhension orale,

* Un E-portfolio pur chaque étudiants,

* Un pré- test des compétences de la compréhension avec un barème de notation,

\section{* Un post-test des compétences de la compréhension un barème de notation.}

\section{(1). Test de placement:}

Le chercheur a préparé un test de placement, pour déterminer le niveau linguistique des étudiants de la troisième année, faculté de pédagogie, université d'Al-Sadat.

Pour élaborer ce test, le chercheur s'est intéressée à :

- Lire des recherches et des documents qui mettent l'accent sur le niveau linguistique du FLE.

- Réviser le Cadre européen commun de référence pour les langues. 
- Réviser les modèles des tests dans la Bibliothèque du Centre Culturel du Français.

- Consulter des références, des questionnaires, des activités, des fiches pédagogiques, des tests et des sites qui évaluent les compétences du FLE. Ces sources sont : Des références comme : Courtillon,(2002), Morlat (2009), et Schmid (2010)

- Des sites d'internet :

- http://www.coe.int/t/dg4/linguistic/source/Framework_FR.pdf

- http://www.bonjourdefrance.com/index/indexappelem.htm

Ce test comprend 16 pages:

- La première page inclut la couverture,

- La deuxième page montre l'objectif, le barème de notation et les consignes.

- La troisième page contient le nom et le prénom de l'apprenant.

- Les autres pages contiennent les quatre parties essentielles de FLE qui sont nécessaires

Le test de placement se compose de 4 parties essentielles du FLE:

- Compréhension orale

- Compréhension des écrits

- Production écrite

- Production orale

\section{Mesure de la validité:}

- Pour réussir à déterminer le niveau linguistique des apprenants, le chercheur a vu important de présenter ce test à des spécialistes du FLE.

- Notons qu'après avoir présenté ce test aux spécialistes, le chercheur a supprimé quelques questions et a modifié d'autres pour le présenter et l'appliquer en sa forme finale.

(2). Fiche d'évaluation des "compétences de la compréhension orale de FLE:

Le chercheur a élaboré une grille d'évaluation appropriée pour déterminer et préciser les compétences de la compréhension orale du FLE chez l'échantillon de la recherche.

Pour élaborer cette liste, le chercheur s'est intéressée à :

- Lire et étudier des recherches, des revues et des articles dans le domaine de la didactique de FLE en général, et du développement de compétence de la compréhension orale en particulier.

- Consulter des références, des documents, des grilles descriptives et des sites d'Internet qui traitent le développement des compétences de la compréhension 
langagière en général, et, en particulier, dans la langue française la compréhension orale.

\section{Cette grille comprend 4 pages:}

- La première page inclut la couverture (titre de la recherche)

- La deuxième page montre 1'objectif et les consignes.

- La troisième page contient les compétences nécessaires de la compréhension orale.

- Notons que La compréhension orale : comprend 11 compétences sousjacentes qui évoquent les compétences nécessaires pour les futuresenseignants.

\section{Mesure de la validité:}

- Conformément aux besoins des apprenants et aux objectifs visés dans les quatre modules de la comprehension mis en ligne sur le dispositif educatif en ligne proposé, le chercheur a inspiré cette grille des competences de la compréhension du CECRL(2001) en mettant en considération le niveau linguistique des membres de l'échantillon (A2) et qui s'adapte à la culture déterminante des mêmes effectifs.

- Pour confirmer la nécessité des compétences visées, le chercheur a vu important de présenter cette liste à des spécialistes de FLE

- Notons qu'après avoir présenté cette grille aux spécialistes, le chercheur a supprimé quelques compétences et a modifié d'autres pour la présenter en sa forme finale.

Forme finale d'une Fiche d'évaluation des "compétences de la compréhension orale du

\section{FLE"}

\section{Fiche des compétences de la compréhension orale}

\section{L'apprenant doit être capable de}

\section{(1): Compréhension globale}

1. Construire une idée générale sur le contenu du texte.

2. Saisir les idées principals

3. Repérer le mot le plus fréquent du texte.

\section{(2): Compréhension détaillée}

4. Saisir les idées secondaires

5. Déterminer le ton d'un texte.

6. Repérer le rythme d'un texte.

7. Inférer les relations (cause, effet et conclusion)

8. Comprendre les interlocuteurs.

\section{(3): Compréhension critique}

9. Inférer l'intention de l'émetteur. 
10. Faire de prédictions.

11. Emettre des jugements et des appréciations personnels.

\section{(3) Pré-Post Test des modules proposés:}

Le test a pour objectif d'évaluer le niveau linguistique des compétences de la compréhension orale du FLE, des futurs-enseignants de la troisième année, section du français, à la faculté de pédagogie d'Al-Sadat à la lueur des axes et des thèmes déjà précis dans les modules proposés en ligne.

\section{Pour élaborer ce test, le chercheur a suivi les démarches suivantes:}

1. Lire et étudier des recherches et des documents qui mettent l'accent sur le rôle des compétences de la compréhension orale en langues étrangères en général, et en langue française en particulier.

2. Consulter des références, des questionnaires, des activités, des fiches pédagogiques, des tests qui évaluent les compétences de la compréhension et des sites d'internet qui s'intéressent à traiter les thèmes et les axes déjà mentionnés aux modules proposés.

\section{Description des items barèmes de notation.}

Chaque test (pré ou poste), se compose de 4 questions divisées en deux parties (auditive et vidéo), la note totale de test est 60 .

Ces questions représentent un amalgame de questions objectives et subjectives qui mesurent l'acquis et la performance de l'échantillon dans les thèmes et les compétences traitées dans les modules proposés.

\section{A. Tests de compréhension auditive :}

- Le chercheur a préparé un test pour chaque chanson (avant et après les modules), après avoir faire un barème et un critère de notation pour les compétences visées.

- Ces tests visent à mesurer la compréhension orale (compréhension orale) des étudiantes à travers une chanson auditive. La note totale pour chaque test $=15$ points.

\section{Les questions sur les tests ont seulement deux types:}

- Répondre aux questions.

- Questions de types (QCM).

B. Tests de compréhension d'une vidéo: Pour la compréhension d'une vidéo, le chercheur a préparé un test pour chaque vidéo, un pré-test et un post-test (avant et après les modules). Ces tests visent à mesurer la compréhension orale (compréhension d'une vidéo) des étudiantes à travers une projection d'une vidéo. La note totale pour chaque test $=15$ points selon un barème et un critère de notation pour les compétences visées.

Mesure de la fidélité du test: On a calculé le coefficient de fidélité en utilisant la méthode "Alpha Cronbach" en ayant recours au logiciel SPSS. On a calculé le coefficient de discrimination pour chaque question afin de savoir la capacité du test à discriminer les questions faciles et difficiles.

Les résultats ont montré que le coefficient d'Alpha Cronbach est 0,62.Cela veut dire que le test est fidèle et applicable.

Mesure de la validité du test :Le calcul de la validité de cohérence interne entre la note totale du test et la note de chaque item 
en calculant le coefficient de corrélation de Guttman Split-Half entre la note de chaque item du test et la note totale du test. Les valeurs sont significatives à un seuil de 0,59.

Durée des tests: Pour déterminer la durée convenable du pré post-test oral, on a demandé à un échantillon d'étudiants de répondre au test de compréhension orale à travers une chanson auditive, test de compréhension orale à travers la projection d'une vidéo. On a calculé la durée prise par l'étudiant qui a fini en premier, et celle prise par l'étudiant qui a terminé son travail en dernier, pour trouver la durée moyenne de répondre au test. Ainsi on a trouvé que la durée globale de l'application du pré post-test est 80 minutes.

\section{(4) : Grille d'évaluation des compétences de la compréhension orale :}

Le chercheur vise à travers de cette grille, à déterminer les compétences les plus nécessaires de la compréhension orale pour les évaluer chez l'échantillon de la recherche.

\section{Sources de la grille:}

1. lire et étudier des recherches, des revues et des articles dans le domaine de la didactique du FLE en général, et du développement de compétence de la compréhension orale en particulier.

2. Consulter des références, des documents, des grilles de vérification et d'évaluation et des sites d'Internet qui traitent le développement des compétences de la compréhension orale dans les langues étrangères en général, et en particulier, dans la langue française visant.

Description des items : Cette grille contient 11 compétences sous-jacentes de la compréhension orale divisées en 3 axes essentiels (compréhension globale compréhension détaillée compréhension critique)

Barèmes de notation: Les barèmes ont été élaborées selon la catégorisation entre (oui ou non) d'après la réalisation de maitrise des apprenants des compétences de la compréhension orale traitées aux modules proposés .La note totale est de 11 Points.

Mesure de la validité: Le chercheur a pris en considération le calcul de la stabilité des correcteurs en ce qui concerne les fiches d'évaluation de compétence de la compréhension orale en utilisant le coefficient de corrélation de "Kendall" entre les notes des correcteurs des fiches d'évaluation de la compréhension orale en ayant recours au logiciel SPSS :

- Coefficient de corrélation $=.78$

- $\quad$ Seuil de signification $=\cdot, \cdots 1$

- Signification au seuil de $(0.05)=$ Significatif

Grille d'évaluation des compétences de la compréhension orale Apprenant :

Module

(

Leçon 
Grille d'évaluation de la compréhension orale

\begin{tabular}{|c|c|c|c|c|}
\hline \multicolumn{5}{|c|}{ éalisation } \\
\hline & \multicolumn{2}{|r|}{ Les activités } & \multirow{2}{*}{ Oui } & \multirow{2}{*}{ Non } \\
\hline 1 & $\begin{array}{l}\text { Compréhension } \\
\text { globale }\end{array}$ & $\begin{array}{l}\text { 1. Construire une idée générale sur le contenu du } \\
\text { texte. }\end{array}$ & & \\
\hline & & 2. Saisir les idées principales. & & \\
\hline & & 3. Repérer le mot le plus fréquent du texte. & & \\
\hline \multirow{5}{*}{2} & \multirow{5}{*}{$\begin{array}{l}\text { Compréhension } \\
\text { détaillée }\end{array}$} & 4. Saisir les idées secondaires. & & \\
\hline & & 5. Déterminer le ton d'un texte. & & \\
\hline & & 6. Repérer le rythme d'un texte. & & \\
\hline & & $\begin{array}{l}\text { 7. Inférer les relations (cause, effet, prédiction et } \\
\text { conclusion) }\end{array}$ & & \\
\hline & & 8. Comprendre les interlocuteurs. & & \\
\hline \multirow{3}{*}{3} & \multirow{3}{*}{$\begin{array}{l}\text { Compréhension } \\
\text { critique }\end{array}$} & 9. Inférer l'intention de l'émetteur. & & \\
\hline & & 10.Faire de prédictions. & & \\
\hline & & $\begin{array}{l}\text { 11. Emettre des jugements et des appréciations } \\
\text { personnels. }\end{array}$ & & \\
\hline
\end{tabular}

\section{(4). E-portfolio pour chaque futur-enseignant:}

L'E-Portfolio est une collection structurée des travaux d'un futur-enseignant et des commentaires qui leur sont attachés, qui fait foi de ses compétences montrant des traces pertinentes de ses réalisations. Les travaux conservés, et parfois affichés, sont sélectionnés en fonction de critères établis par le futur-enseignant et par l'enseignant. (Berthiaume, D.2015:55)

E-portfolio se compose de plusieurs documents:

* Des activités variées selon les modules proposés d'apprentissage à la lueur de la collaboration en ligne et pendant le déroulement de chaque séance ou activité en 
ayant recours à la technologie afin de les grouper en une seule programmation.

* Outils pour la collecte des résultats des futurs-enseignants afin de connaître leur progression :

- Pré-test mesurant les compétences de la compréhension orale à travers un document auditif.

- Pré-test mesurant les compétences de la compréhension orale à travers un document d'une vidéo.

- Post-test mesurant les compétences de la compréhension orale à travers un document auditif.

- Post-test mesurant les compétences de la compréhension orale à travers un document d'une vidéo.

\section{(Axe 2). Matériel de l'expérimentation de la recherche}

Dans cet axe, le chercheur va proposer des modules dont l'objectif est l'acquisition et le développement des compétences de la compréhension de FLE (orale). Ces modules sont élaborés à destination des étudiants de la troisième année, section du français, faculté de Pédagogie, université d'Al-Sadat.

Le chercheur a essayé de respecter une progression dans la présentation du sujet selon la logique du facile au difficile, mais aussi selon les besoins concrets des apprenants. L'acquisition des compétences de la compréhension de FLE passe dans les modules proposés à travers le développement de toutes les compétences.

Dans le programme proposé, le futur-enseignant peut suivre trois modules interactifs en ligne autour du thème de la compréhension orale pour développer quelques compétences orales, lorsqu'on part écouter, et visionner des textes, et des activités différentes en améliorant quelques compétences de la compréhension (orale), tout au long des modules.

Pour élaborer le dispositif éducatif proposé, le chercheur a organisé une conception du dispositif éducatif autour de 5 phases essentielles comme suit :

- Analyse

- Conception

- Organisation du dispositif mis en ligne

- Développement

- Mise en auvre

- Evaluation 


\begin{tabular}{|c|c|}
\hline 1. Analyse & $\begin{array}{l}\text { Choisir l'échantillon ; } \\
\text { analyser ses besoins ; } \\
\text { analyser ses prérequis (pour mesurer leurs compétences de la compréhension) } \\
\text { analyser des thèmes (pour identifier et décrire le contenu du cours) } \\
\text { analyser des tâches (pour identifier les tâches que les apprenants doivent appre } \\
\text { lécrire le contenu. }\end{array}$ \\
\hline 2. Conception & $\begin{array}{l}\text { Déterminer les objectifs et les compétences à acquérir ; } \\
\text { Déterminer le séquençage pour définir l'ordre dans lequel les objectifs doive } \\
\text { atteints : (Réflexion sur les différentes compétences de la compréhension); } \\
\text { Déterminer les thèmes et les tâches pour réaliser les objectifs ; } \\
\text { déterminer les matériels, les supports (multimédias, documents, vidéo, et audid } \\
\text { les ressources pédagogiques complémentaires. } \\
\text { décrire la structure du contenu (son organisation en modules, leçons et activité } \\
\text { pétenter les objectifs d'apprentissage associés à chaque module; } \\
\text { déterminer les modalités d'apprentissage (à distance) ; } \\
\text { processus métacognitifs }\end{array}$ \\
\hline 1. development & $\begin{array}{l}\text { Elaborer le contenu ; } \\
\text { Préparer un guide de l'enseignant en déterminant son rôle et celui de l'appren } \\
\text { Réviser et évaluer le contenu didactique au niveau de la langue ; } \\
\text { installer le dispositif éducatif sur la plateforme; } \\
\text { Evaluer le dispositif éducatif au niveau technologique pour assurer sa structur } \\
\text { et l'ergonomie du site ; } \\
\text { Déterminer les noms d'utilisateurs et les mots de passe pour chaque étudiar } \\
\text { accéder au site https://sites.google.com/view/collaboratifeu/accueil ; }\end{array}$ \\
\hline $\begin{array}{l}\text { 4. Mise en } \\
\text { œuvre }\end{array}$ & $\begin{array}{l}\text { Mettre en ligne le dispositif éducatif ; } \\
\text { Donner le nom d'utilisateur et le mot de passe pour chaque étudiant } \\
\text { Expliquer aux étudiants comment accéder aux cours et comment passer d'une } \\
\text { à l'autre ; }\end{array}$ \\
\hline 5. Evaluation & $\begin{array}{l}\text { Evaluer les apprenants tout au long des modules proposés pour mesurer leur n } \\
\text { des compétences de la compréhension (évaluation formative); } \\
\text { Evaluer la réalisation des objectifs de chaque module pour orienter les apprenan } \\
\text { un autre module de formation ; (évaluation sommative); } \\
\text { Evaluer les processus métacognitifs des apprenants dans les compétences } \\
\text { compréhension; } \\
\text { Evaluer la réaction des apprenants à travers une fiche intitulée "Ré } \\
\text { métacognitive" qui est présentée à la fin de chaque module ; } \\
\text { analyser les résultats qualitativement et quantitativement ; } \\
\text { discuter les résultats en présentant les suggestions et les recommandations, sel } \\
\text { résultats, pour des recherches prochaines. }\end{array}$ \\
\hline
\end{tabular}

Le chercheur vise à extraire et à analyser les résultats de ce travail en suivant la méthode mixte qui combine des données/méthodes quantitatives et qualitatives. 
Dans cette optique, la recherche actuelle a recouru au modèle de "La triangulation" comme un des modèles de méthodes mixtes. Il s'agit, comme l'indique Vianin (2011), d'obtenir des données différentes mais complémentaires sur un même sujet afin de mieux comprendre le problème de recherche. D'après ce modèle, Bollecker et Nobre (2015) supposent que le chercheur utilise plusieurs sources d'informations (orales, écrites), différentes méthodes d'enquête (questionnaires, observations) et divers informateurs.

\section{Axe (1) Pour l'analyse quantitative:}

L'hypothèse nulle est: "Il n'y a pas de différences statistiquement significatives entre les moyennes des notes des futurs-enseignants aux pré-post tests des compétences de la compréhension orale.»

Le chercheur a analysé les résultats de l'échantillon du pré-post-test avant et après l'expérimentation .Puis, il a calculé la moyenne et les écarts types pour le pré-post test, et le niveau de signification statistique entre la moyenne des notes de l'échantillon au pré-test des compétences de la compréhension orale et la moyenne des étudiants de leurs notes au post-test .

Dans le tableau n. ${ }^{\circ}(1)$ et les diagrammes $n .^{\circ}$ (1) suivants, le chercheur indique les résultats des étudiants sur le pré-post test des compétences de la compréhension orale pour comparer leurs performances.

Tableau n. ${ }^{\circ}$ (1): Résultat de l'application du test de Wilcoxon pour comparer les performances des étudiants sur le pré-post test des compétences de la compréhension orale.

\begin{tabular}{|c|c|c|c|c|c|c|c|c|c|}
\hline 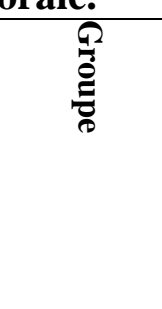 & 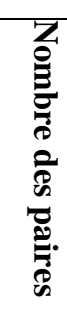 & 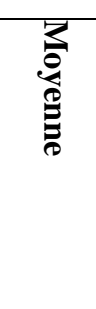 & 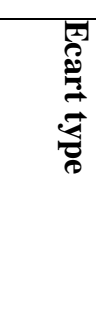 & 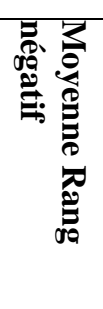 & 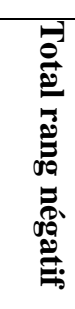 & 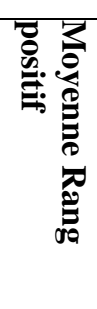 & 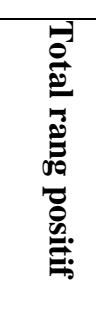 & 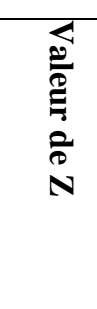 & 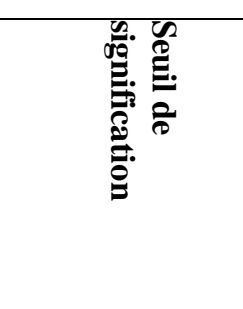 \\
\hline $\begin{array}{l}\text { Oral-pré } \\
\text { Oral-post }\end{array}$ & 12 & $\begin{array}{l}11.41 \\
28.66\end{array}$ & $\begin{array}{l}1.83 \\
0.778\end{array}$ & 0.00 & 0.00 & 6.50 & 78.00 & 3.075 & Significatif 0.02 \\
\hline
\end{tabular}




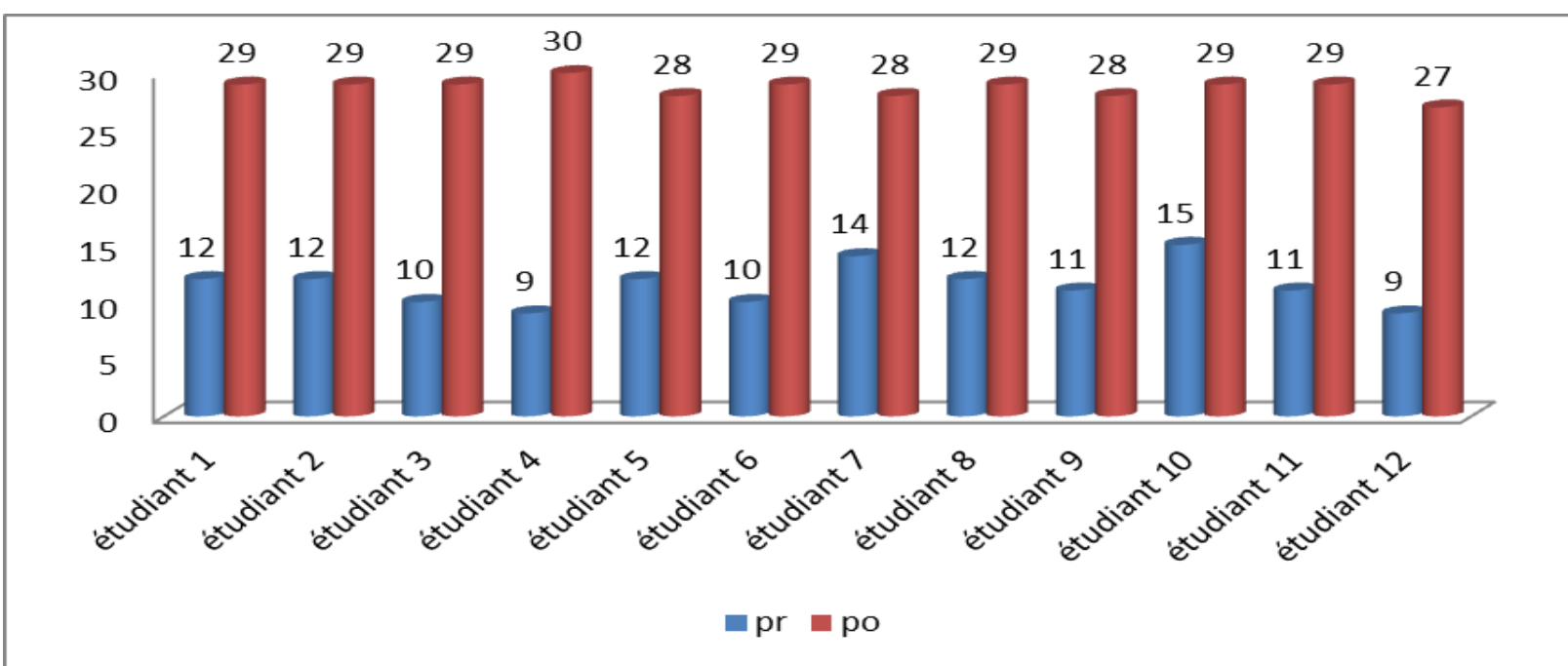

Diagramme n. ${ }^{\circ}$ (1) : Notes de l'échantillon sur le pré-post application du test des compétences de la compréhension orale

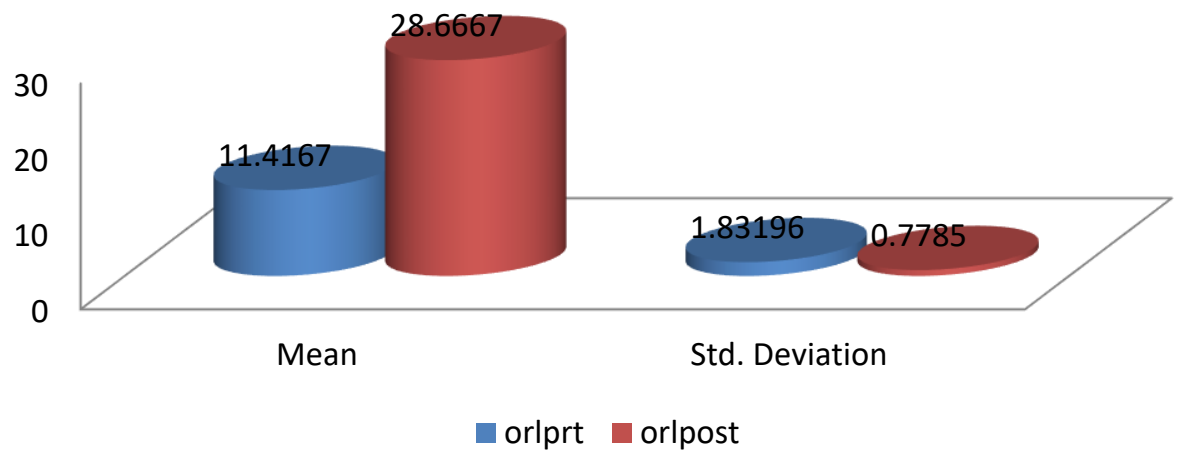

Diagramme n. ${ }^{\circ}(2)$ : Notes totales de l'échantillon sur le pré-post application du test des compétences de la compréhension orale.

Selon le tableau $n^{\circ}$ (1) et les diagrammes $n .^{\circ}$ (1 et 2), les résultats montrent qu'il y a de différences significatives au seuil de $(\boldsymbol{\alpha} \leq \mathbf{0 . 0 2})$, dans la compétence de la compréhension orale comme une des compétences de la compréhension du FLE au pré-post tests de l'échantillon où la valeur de Z est (3.075) et elle est significative au seuil de (0.02)

Alors, on peut dire que le dispositif éducatif proposé a un effet essentiel sur le développement des compétences de la compréhension orale. En outre, le chercheur croit que l'amélioration des apprenants dans les compétences de la compréhension orale revient à des facteurs comme: avoir de motivation d'achèvement chez les apprenants, les modules en ligne prennent du tout en considération l'entrainement des apprenants sur la compétence de compréhension orale, les processus mentaux des apprenants qu'ils font pendant le processus du visionnage sont réguliers, donner l'occasion aux apprenants à s'auto-enseigner, s'auto-corriger et travailler ensemble, prendre le temps pour préparer, planifier et réviser leurs réponses, diversifier les activités de la compréhension orale, impliquer les apprenants dans l'évaluation de leurs propres productions et recourir aux liens, aux sites et aux documents complémentaires d'enrichissement pour approfondir leurs connaissance, les 
enregistrements des apprenants pour vérifier leur compréhension, la diversité des activités d'entrainement sur les compétences orales d'une manière simultanée ont un effet sur le développement de compétences la compréhension orale.

L'hypothèse dirigéeest: Il y a de différences statistiquement significatives entre les moyennes des notes des apprenants aux pré-post tests des compétences de la compréhension orale du FLE en faveur du post-test.

Le chercheur a analysé les résultats de l'échantillon du pré-post-test avant et après l'expérimentation .Puis, il a calculé la moyenne et les écarts types pour le pré-post test, et le niveau de signification statistique entre la moyenne des notes de l'échantillon au pré-test des compétences de la compréhension orale du FLE et la moyenne des étudiants de leurs notes au post-test .

Tableau n. ${ }^{\circ}$ (2): Résultat de l'application du test de Wilcoxon pour comparer les performances des étudiants sur le pré-post test des compétences de la compréhension orale du FLE.

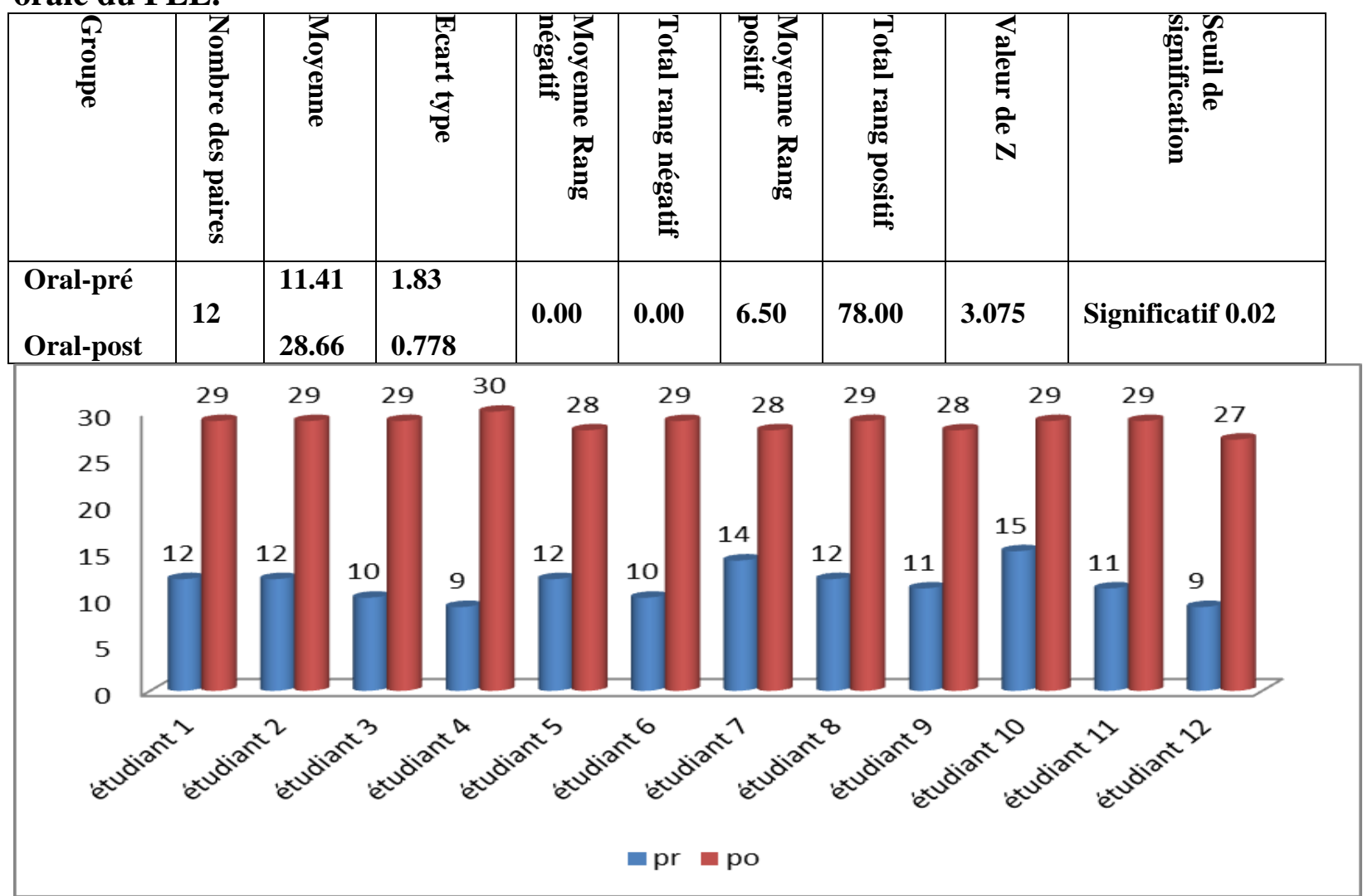

Diagramme n. ${ }^{0}(3)$ : Notes de l'échantillon sur le pré-post application du test des compétences de la compréhension du FLE 


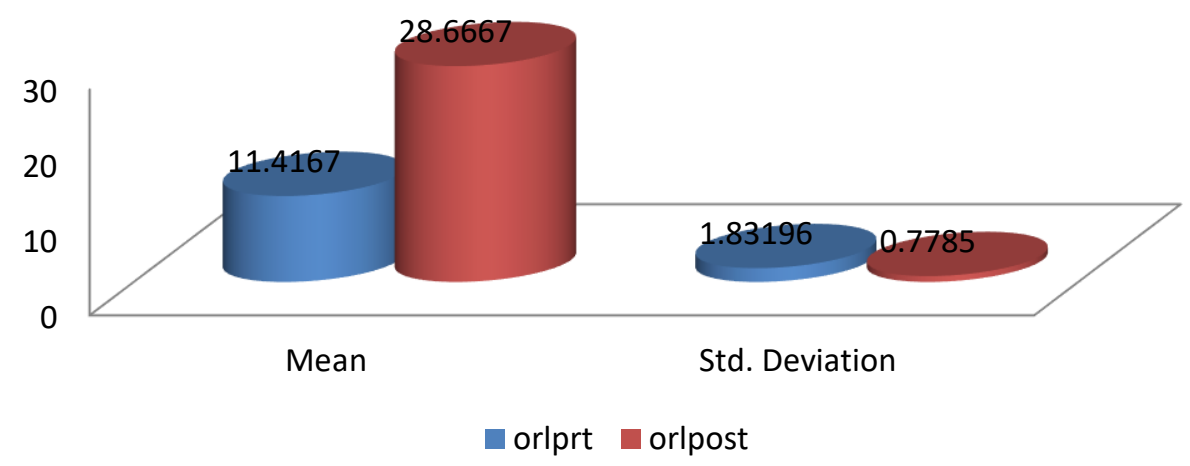

Diagramme n. ${ }^{\circ}(4)$ : Notes totales de l'échantillon sur le pré-post application du test des compétences de la compréhension du FLE

Selon le tableau $n .^{\circ}(2)$ et les diagrammes $n .^{\circ}$ (3et 4) précédents, on confirme qu'il y a des différences significatives au seuil de (0.02) au niveau des compétences de la compréhension orale du $\boldsymbol{F L E}$ entre le pré-post-test de l'échantillon en faveur du posttest.Alors, la valeur de Z est significative au seuil de (3.075), selon le test de Willcoxon. $C$ 'est-à-dire que $100 \%$ de la dissemblance des notes des apprenants sur le test revient au dispositif éducatif et les modules proposés.

Donc, on peut dire que le dispositif éducatif proposé a un grand effet observable sur le développement des compétences de la compréhension du FLE pour tous les étudiants. Discussion des résultats de la performance des apprenants dans la compétence de compréhension orale :

En effet, la compétence de la compréhension orale est la compétence la plus traitée et la plus accentuée dans le dispositif éducatif parce que les apprenants ont besoin d'établir des notions de base, de comprendre, d'identifier les sens, et par conséquent, de comprendre le texte. Cette compétence est divisée en 3 modules, chaque module comprend 2 objets et 2 bilans sur l'oral dans lesquelles les apprenants doivent acquérir des compétences de la compréhension orale.

A travers les tableaux n. ${ }^{\circ}(3),{ }^{\circ}{ }^{\circ}$ (4)et n. ${ }^{\circ}(5)$, et les diagrammes $n . .^{\circ}(13),{ }^{\circ}{ }^{\circ}(14)$, n. ${ }^{\circ}$ (15), n. ${ }^{\circ}$ (16), et n. $^{\circ}(\mathbf{1 7})$, suivants, le chercheur présente les notes des apprenants dans le pré-post test de la compétence de compréhension orale en montrant leur pourcentage dans cette compétence.

Tableau n. ${ }^{\circ}$ (3): Notes et pourcentages des apprenants au pré-post tests des compétences de la compréhension orale.

\begin{tabular}{|l|c|c|c|c|}
\hline Apprenant & pré-test & pourcentage & post-test & pourcentage \\
\hline apprenant 1 & 12 & $40 \%$ & 29 & $96.66 \%$ \\
\hline apprenant 2 & 12 & $40 \%$ & 29 & $96.66 \%$ \\
\hline apprenant 3 & 10 & $33.33 \%$ & 29 & $96.66 \%$ \\
\hline apprenant 4 & 9 & $30 \%$ & 30 & $100 \%$ \\
\hline apprenant 5 & 12 & $40 \%$ & 28 & $93.33 \%$ \\
\hline apprenant 6 & 10 & $30.33 \%$ & 29 & $96.66 \%$ \\
\hline apprenant 7 & 14 & $46.66 \%$ & 28 & $93.33 \%$ \\
\hline apprenant 8 & 12 & $40 \%$ & 29 & $96.66 \%$ \\
\hline
\end{tabular}




\begin{tabular}{|l|c|c|c|c|}
\hline apprenant 9 & 11 & $36.33 \%$ & 28 & $93.33 \%$ \\
\hline apprenant 10 & 15 & $50 \%$ & 29 & $96.66 \%$ \\
\hline apprenant 11 & 11 & $36.33 \%$ & 29 & $96.66 \%$ \\
\hline apprenant 12 & 9 & $30 \%$ & 27 & $90 \%$ \\
\hline
\end{tabular}

Tableau n. ${ }^{\circ}(4):$ Pourcentages des apprenants aux modules des compétences de la compréhension orale.

\begin{tabular}{|c|c|c|c|}
\hline Module & \multicolumn{3}{|c|}{ Compétence de la compréhension orale } \\
\hline enant & $\begin{array}{c}\text { Module } \\
1\end{array}$ & $\begin{array}{c}\text { Module } \\
2\end{array}$ & $\begin{array}{c}\text { Module } \\
3\end{array}$ \\
\hline apprenant (1) & $81 \%$ & $83.33 \%$ & $98.33 \%$ \\
\hline apprenant (2) & $78 \%$ & $86.66 \%$ & $98.33 \%$ \\
\hline apprenant (3) & $76 \%$ & $86.66 \%$ & $98.33 \%$ \\
\hline apprenant (4) & $80 \%$ & $82 \%$ & $96.66 \%$ \\
\hline apprenant (5) & $75 \%$ & $85 \%$ & $98.66 \%$ \\
\hline apprenant (6) & $68 \%$ & $86.66 \%$ & $98.66 \%$ \\
\hline apprenant (7) & $75 \%$ & $81.66 \%$ & $98.66 \%$ \\
\hline apprenant (8) & $80 \%$ & $81.66 \%$ & $96.66 \%$ \\
\hline apprenant (9) & $78 \%$ & $81.66 \%$ & $96.66 \%$ \\
\hline apprenant (10) & $75 \%$ & $86.66 \%$ & $96.66 \%$ \\
\hline apprenant (11) & $76 \%$ & $85 \%$ & $100 \%$ \\
\hline apprenant (12) & $61.66 \%$ & $76.66 \%$ & $90 \%$ \\
\hline
\end{tabular}

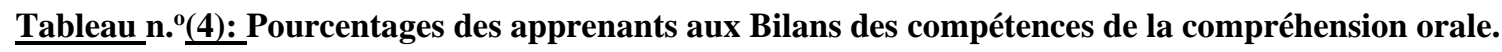

\begin{tabular}{|l|c|c|c|}
\hline & \multicolumn{3}{|c|}{ Compétence de la compréhension orale } \\
\hline & Bilan & Bilan & Bilan \\
\hline Apprenants & 1 & 2 & 3 \\
\hline apprenant (1) & $78 \%$ & $90 \%$ & $93.33 \%$ \\
\hline apprenant (2) & $76 \%$ & $90 \%$ & $93.33 \%$ \\
\hline apprenant (3) & $73.33 \%$ & $88.33 \%$ & $93.33 \%$ \\
\hline apprenant (4) & $66.66 \%$ & $91.66 \%$ & $93.33 \%$ \\
\hline apprenant (5) & $75 \%$ & $90 \%$ & $95 \%$ \\
\hline apprenant (6) & $73.33 \%$ & $90 \%$ & $95 \%$ \\
\hline apprenant (7) & $66.66 \%$ & $91.66 \%$ & $93.33 \%$ \\
\hline apprenant (8) & $75 \%$ & $88.33 \%$ & $93.33 \%$ \\
\hline apprenant (9) & $71.33 \%$ & $90 \%$ & $93.33 \%$ \\
\hline apprenant (10) & $73.33 \%$ & $88.33 \%$ & $95 \%$ \\
\hline apprenant (11) & $73.33 \%$ & $86.66 \%$ & $93.33 \%$ \\
\hline apprenant (12) & $65 \%$ & $83.33 \%$ & $90 \%$ \\
\hline
\end{tabular}


Research Title: Effet de l'apprentissage collaboratif en ligne pour améliorer la compréhension orale du FLE chez les futurs-

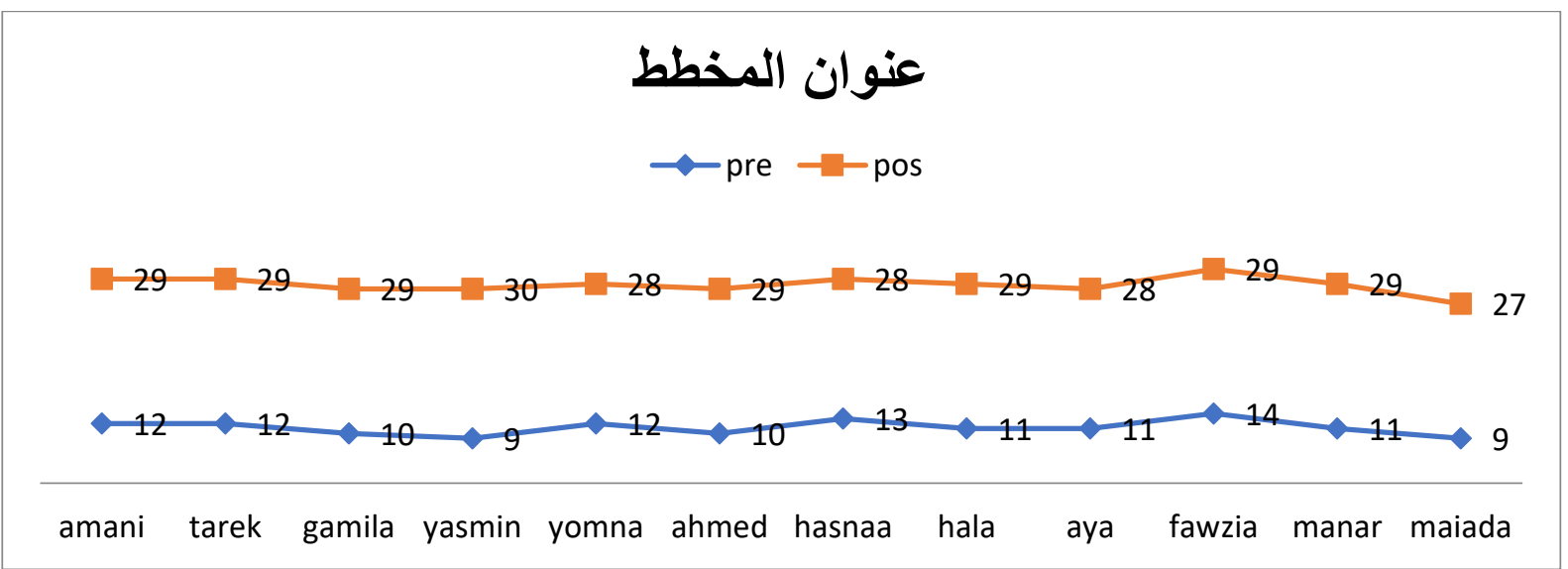

Diagramme n. ${ }^{\circ}(5)$ : Notes des apprenants de l'échantillon au pré-post application du test des compétences de la compréhension orale.

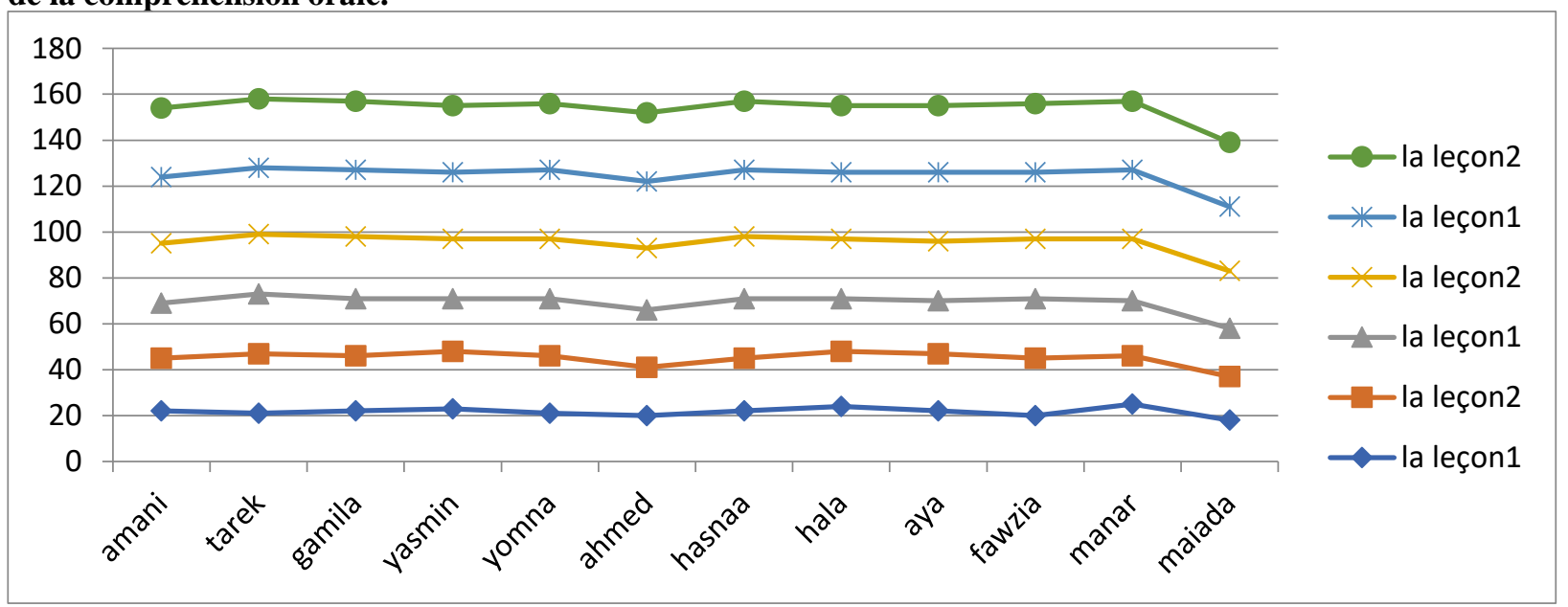

Diagramme n. ${ }^{0}(6)$ : Notes des apprenants aux modules des compétences de la compréhension orale.

\section{عنوان المخطط}

$\checkmark$ module 1 Évaluation1 - module 1 Évaluation $2-$ module 2 Évaluation1

$\leftarrow$ module 2 Évaluation 2 * module 3 Évaluation1 $\multimap$ module 3 Évaluation2

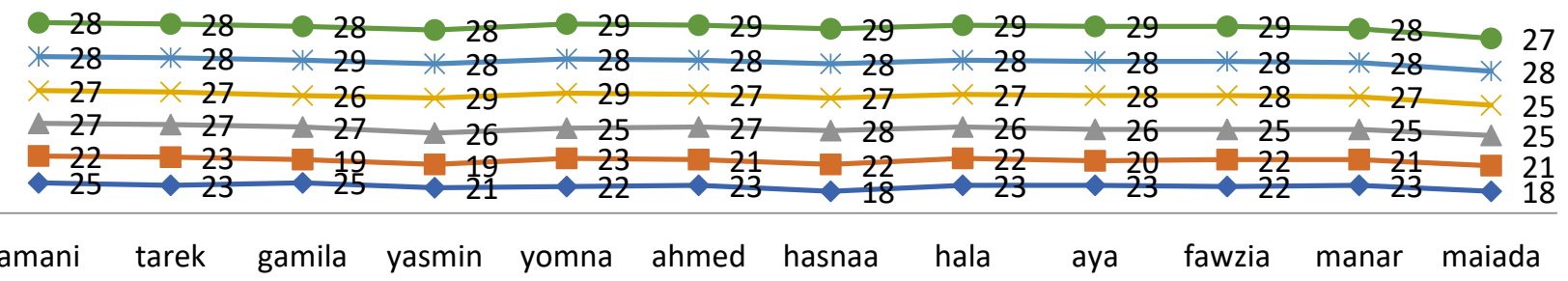

Diagramme n. ${ }^{\circ}(17)$ : Notes des apprenants aux Bilans des compétences de la compréhension orale.

- Pour le pré-test, le chercheur a marqué que cette compétence est la plus ignorée et la plus ambigue de la part des apprenants. $\mathbf{7 0} \%$ des apprenants n'ont pas pu répondre aux questions qui mesurent les compétences-adjointes. Et les autres ont essayé de répondre mais d'une manière vague, incorrecte et incompréhensible. 
Par exemple, la plupart des apprenants ne comprennent pas ce qu'on veut de "Déterminer le ton d'une chanson, Inférer l'intention de l'émetteur, Faire de prédictions...etc»

En effet le chercheur met en évidence que les résultats du pré-test, comme il a indiqué précédemment, proviennent à l'absence de la conscience et de la formation dans la compétence de compréhension du FLE chez les apprenants. Or, leurs compétences communicatives insuffisantes et faibles représentent un obstacle pour s'exprimer d'une manière évidente et correcte.

- Alors qu'au post-test, aux modules et aux bilans, leurs résultats sont meilleurs en réalisant un progrès assez élevé. En général, ces résultats qui réalisent peu à peu un succès après chaque module, révèlent l'efficacité des activités d'apprentissage visées dans les modules interactifs basés sur l'apprentissage collaboratif en ligne. Tandis que les notes de l'apprenante $n .^{\circ}(\mathbf{1 2})$ a commencé peu d'augmenter, car le dernier apprenant est toujours occupée par son bébé et a beaucoup de difficultés au réseau de l'internet dans son village.

Il est à noter que les résultats du post-test s'accorde avec l'avis de France (2011), Henri et Cayrol (2001) qui montrent que la mise en œuvre d'une apprentissage collaboratif en ligne dans l'enseignement des langues étrangères suppose le passage d'un rôle traditionnel vers un nouveau rôle de médiateur positif dont l'un des objectifs majeurs est de développer chez les apprenants des compétences de la compréhension du FLE.

Selon l'interprétation qualitative du chercheur de chaque compétence attendue de la compréhension orale du FLE, on a constaté que la performance générale de la plupart des apprenants s'est développée pendant les modules du dispositif éducatif proposé.

Le chercheur a observé que les apprenantes n'ont pas essayé d'utiliser leurs propres mots et leurs connaissances antérieures pour répondre et justifier leurs points de vue. Habituellement, ils ont recourt à tirer, à copier et à coller les informations requises sans fonctionner leur connaissances antérieures pour s'exprimer et interpréter leurs réponses. De même, ils n'ont pas fait attention à s'auto corriger les réponses produites en fonction des consignes en négligeant souvent à recourir à leur tâches et approfondir leurs connaissances.

En grand, le chercheur a remarqué que les apprenants affrontent beaucoup de difficultés à s'exprimer en français, à construire des phrases correctes, à connaitre le mot convenable au bon contexte, à prononcer correctement les sons et à exposer des idées claires et organisées.

\section{Conséquences pédagogiques de la recherche et ses recommandations}

Le chercheur voit qu'il est nécessaire d'aborder les conséquences pédagogiques liées aux résultats de l'étude à la lumière de trois dimensions représentées dans :

Les conséquences pédagogiques théoriques (qui s'attachent au profit des études, des recherches et des références théoriques pertinentes à l'objet de la recherche)

Les conséquences pédagogiques empiriques (qui s'attachent à l'observation et aux facteurs effectifs du développement des compétences de la compréhension orale 
Les conséquences pédagogiques méthodologiques (qui s'attachent à la méthodologie utilisée dans la recherche pour réaliser ses objectifs)

Axe (1): Les conséquences pédagogiques théoriques :

1. Importance de l'apprentissage de la compréhension orale dans la classe du FLE : La compréhension orale du FLE a pour but de communiquer, construire la personnalité, et vivre ensemble, apprendre des conceptions, des représentations, construire la pensée, et réfléchir sur le langage.

2. Importance de l'utilisation de l'apprentissage collaboratif en ligne dans l'enseignement /apprentissage la compréhension du FLE : le chercheur a choisi cette stratégie comme une stratégie principale à côté d'autres outils déjà mentionnées qui est, parmi ces outils, la plus convenable avec la nature de cette recherche et son objectif général qui se réside en: développer quelques compétences de la compréhension du FLE à l'aide des TICE.

3. Importance des TICE pour enseigner/apprendre les compétences de la compréhension du FLE : l'intégration des Technologies de l'Information et de la Communication (TICE) dans la didactique des langues étrangères nous offre aujourd'hui la possibilité de mettre en place un dispositif d'apprentissage où les apprenants travaillent en prenant en charge leur apprentissage.

Axe (2): Les conséquences pédagogiques empiriques :

1. Sensibiliser les apprenants au rôle de la compréhension du FLE et ses compétences adjointes, pour prendre conscience de leur importance dans l'apprentissage: D'après les résultats des apprenants dans cette recherche actuelle:84\% des apprenant sont marqué leur désir d'apprendre sur l'Internet afin d'éviter les difficultés langagières.

2. Considérer l'acquisition quelques compétences de la compréhension du FLE comme premier pas pour apprendre la langue : Les résultats de cette recherche a indiqué que $85 \%$ des apprenants ont marqué qu'ils sont fières d'apprendre et d'enseigner d'autres compétences langagières.

Axe (3): les conséquences méthodologiques: le chercheur a utilisé les deux formes de données en même temps et elles sont ensuite intégrées dans l'interprétation des résultats globaux .Le chercheur a présenté les résultats en deux temps. Dans un premier temps, elle a commenté les données quantitatives par l'intermédiaire de la formulation; dans un second temps, elle a fait une analyse et une interprétation approfondie des données.

De tout ce qui précède, le chercheur peut proposer les suggestions suivantes pour des études prochaines:

1. Profiter de l'apprentissage collaboratif en ligne pour une formation continue sur différentes activités linguistiques, ainsi que pour former des enseignants de 
français sur différentes compétences linguistiques, car cela se fait de manière sécurisée, facile et peu coûteuse.

2. Profiter de l'apprentissage collaboratif en ligne pour développer les compétences écrites chez les lycéens.

3. Entrainer les futurs enseignants d'exploiter les nombreux outils des TICE pour maitriser la langue française.

4. Attirer l'attention sur l'efficacité de l'apprentissage hybride pour développer le FLE.

5. Mettre en évidence l'importance de l'intégration des TICE dans l'enseignement du FLE.

6. Mettre l'accent sur l'apprentissage numérique du FLE.

7. Inviter à perfectionner les compétences de la compréhension orale et celles de la compréhension écrite du FLE chez les apprenants universitaires.

\section{BIBLIOGRAPHIE}

\section{(A). Bibliographie en langue francaise :}

- Allaire, S., \& Lusignan, G. (2019):" Enseigner et apprendre en réseau": collaborer entre écoles distantes à l'aide des TIC. Anjou: CEC.

- Arpin, L. et Carpa, L. (2010): "L’apprentissage par projets": Montréal: Chenelière/ McGraw-Hill.

- Berthiaume, D., Daele, A. (2015):" Faire appel au portfolio pour évaluer les apprentissages des étudiant-e-s?". Disponible en ligne sur:http://www.unil.ch/webdav/site/cse/shared/brochures/memento_portfolio.pdf

- Bollecker et Nobre (2015):"Pourquoi le contrôle de gestion à l'université est-il un échec?" un exemple d'évaluation diagnostique des processus cognitifs». Bruxelles: De Boeck.

- Cadre européen commun de référence pour les langues (2013):" apprendre, enseigner", évaluer. Disponible en ligne sur <http://www.coe.int/t/dg4/linguistic/Source/ Framework_fr.pdf>, p. 54.

- CECRL (2001):" les descripteurs généraux de la compréhension (orale et écrite) suivant. les niveaux déterminés dans le cadre commun de référence pour les langues

- Cornaire, C. (2006): "la compréhension orale. Paris": CLE. Internationale.

- Courtillon, J. (2002):" pour élaborer un cours de FLE". Hachette, Paris.

- Duquesnoy, M. (2018). :" avantages la technologie en classe \& 18 façons de l'intégrer dans ses cours.Disponible sur: https://portaileduc.net/website/9avantages-la-technologie-en-classe-18-facons-de-lintegrer-dans-ses-cours/

- Dussault-Désy, L. (2010):" Choisir l'apprentissage collaboratif comme outil d'intervention en classe de rédaction technique", Essai présenté pour l'obtention d'une Maîtrise en enseignement.Sherbrooke : Université de Sherbrooke.

- Ferroukhi, K. (2009):" La compréhension orale et les stratégies d'écoute des élèves apprenant le français en 2ème année moyenne en Algérie", Université de Blida. Disponible en ligne sur: http://gerflint.fr/Base/Algerie4/ferroukhi.pdf.Consulté le 3 
- franc. P .(2018):" compréhension orale et apprentissage de la langue, magazine électronique", franc .parler, Disponible en ligne sur http://franc.Parler,org.// .

- France, E. (2011):" TIC et formation des enseignants du fondamental en Haïti": barrières et facteurs facilitants. Thèse de doctorat, Université de Montréal.

- Guichon, N. (2015):" Vers l'intégration des TIC dans l'enseignement des langues". Paris : Didier.

- Henri, F, Lundgren - Cayrol, K. (2001):" Apprentissage collaboratif à distance pour comprendre ". Presses de l'université du Québec.

- Henri, F, Lundgren - Cayrol, K. (2003):" Apprentissage collaboratif à distance. Pour comprendre et concevoir des environnements d'apprentissage virtuels". Sainte-Foy : Presses de l'Université du Québec

- Henri, F, Lundgren - Cayrol, K. (2008):" Apprentissage collaboratif à distance. Pour comprendre et concevoir des environnements d'apprentissage virtuels". Sainte-Foy : Presses de l'Université du Québec.

- Lhote,F.(2005):" Enseigner l'oral en interaction",L'arts,Hachette.

- Mackay, W. (2002) : " Principes de didactique analytique", Paris, Didier.

- Marchi, Brigitte Urbano (2010):"Le «savoir-être» pour la formation initiale dans le contexte du CECRL": la prise en compte du handicap par le biais du «slam», CiDd , II congres International de didactiques 2010 , L'activité de l'enseignant : Intervention, Innovation ,Recherche, Girona les 3, 4,5 et 6 février.

- Michelas, A. (2019):" Caractérisation phonétique et phonologique du syntagme intermédiaire en français de la production à la perception". Thèse de doctorat, Université d'Aix-Marseille.

- SPRINGER, C., Koenig-WIŚNIEWSKA, A. (2019):" Du journal intime aux réseaux sociaux", en Le Français dans le monde, $\mathrm{n}^{\circ}$ 351, mai-juin, Paris, CLE International Disponible en ligne sur,http://www.fdlm.org/fle/article/351/springer.php.

- Vianin,P. (2011):" Neurosciences cognitives et pédagogie spécialisé": un exemple d'évaluation diagnostique des processus cognitifs». Bruxelles: De Boeck.

- (B). Bibliographie en langue anglaise :

- Burge, E. (2014):" Learning in computer conference contexts": The learner's perspective. Journal of Distance Education, 9(1), 19-43.

- Schimidt-Rinehart, B. C. (2009): "The effects of topic familiarity on second language listening comprehension", The Modern Language Journal, 78.

- Schrage, M. (2011):" Shared minds the new technologies of collaboration". New York: Random House.

- Vendergrift (2012):" facilating second language listening comprehension acquiring successful strategies". ELT journal 53 (3). 